\title{
The beauty of the small
}

\author{
Plant biology has a long history in helping to illuminate the most detailed workings of living organisms. \\ This tradition is amply represented by a trio of structures appearing this month.
}

In this issue of Nature Plants, there are three Articles reporting the atomic structures of plant proteins ${ }^{1-3}$. This slightly surprised us; enough that we have joked about temporarily changing the journal's name to reflect this unusual concentration of structural papers. It should not have done. The nanoscale workings of the machinery of plants are no less important than those of any other division of life. In fact, 'plant structural biology' has been at the heart of a number of fundamental advances in biology.

Consider cell theory. In the seventeenth century, Robert Hooke was using the cuttingedge technology of the time, the microscope, to look at the natural world. He described his observations in his 1665 book Micrographia, which arose from a commission by Charles II of England (by way of Christopher Wren) to perform microscopic studies of insects. Hooke took upon himself a wider remit, looking at other materials, including thin slices of cork. In these he saw empty spaces surrounded by solid walls, which he named 'cells'. Not content with just observing, Hooke used his $50 \times$ magnifying microscope to calculate that there would be almost 1,260 million cells in a cubic inch.

By the early twentieth century, the first studies of biological molecules using $\mathrm{X}$-ray diffraction were being undertaken, including ones on cotton. In the 1920s, two German chemists, Kurt Heinrich Meyer and Herman Francis Mark, obtained diffraction patterns from cotton and used these to support the theory that such fibres consisted of long macromolecules of regularly repeating, covalently bonded subunits. For cotton, or rather the cellulose that is its major component, the subunit consisted of two glucose molecules. It is difficult to overestimate the influence of Meyer and Mark and their work on the development of structural biology. Their X-ray pictures inspired William Astbury in Leeds, UK, to conclude that his diffraction patterns from keratin demonstrated that fibrous proteins were also polymers, but of amino acids rather than sugars. Mark also taught X-ray diffraction to Linus Pauling and Max Perutz, both of who subsequently won Nobel prizes for different aspects of structural biology.

Despite this early importance of plant structures, the focus of early molecular and structural biology was much more on animal proteins. Only around $4 \%$ of the yearly entries into the Protein Data Bank (http://www.rcsb. org/pdb/) have a plant origin.

Nevertheless, there are many interesting structures and unique research questions to be answered. For example, Hirano et al. ${ }^{1}$ have investigated the interactions that lie at the heart of root patterning by determining the structure of a heterodimer of SCARECROW (SCR) and SHORT-ROOT (SHR), both on its own and when bound to the transcription factor JACKDAW (JKD). These specific interactions are important for determining cell fates in the developing root, but SCR and SHR are also representatives of a large family of transcriptional regulators known as GRAS proteins due to their homologous GRAS domains. There are 33 such proteins encoded in the Arabidopsis genome and twice that number in rice, providing the potential for a diversity of functions through their ability to promiscuously dimerize. That any of the possible partnerships can associate with various members of the BIRD family of transcription factors, of which JKD is a representative, leads to a highly flexible system of developmental control unique to plants.

Wang et al. ${ }^{2}$ also look at a plant-specific process: chloroplast division. Chloroplasts and other plastids are double-membrane intracellular organelles that must divide autonomously in order to maintain their numbers in daughter cells during cell division. This is achieved by the action of two contractile rings, one on the interior of the inner envelope membrane, formed by the protein FtsZ, and one on the cytosolic side of the outer envelope, assembled from ARC5. For successful chloroplast division, the activities of these two rings must be co-ordinated. This is achieved by the proteins PDV2 and ARC6, which span the outer and inner envelope membranes, respectively, and reach across the intermembrane space to bind with each other. This interaction must be relatively weak to establish dynamic communication between the two proteins, making the task of imaging their complex doubly difficult. Wang et al. solved this particular problem by linking the interacting domains of PDV2 and ARC6 together with a flexible tether.

Such technical difficulties occur with systems from all branches of life, but plants provide one of the greatest challenges in structural biology: the photosystems at the heart of photosynthesis. Not only are the photosystems integral to the chloroplast thylakoid membrane, which immediately causes problems for conventional crystallography. They are also composed of multiple protein subunits and around a hundred or more cofactors, all of which are needed to understand photosystem functioning. Worse yet, they have a dynamic relationship with additional components, including the hardly less complicated lightharvesting complexes, forming and reforming supercomplexes dependent on exact conditions in the chloroplast.

As long ago as 1988, Johann Deisenhofer, Robert Huber and Hartmut Michel were awarded the Nobel prize for chemistry for their determination of the structure of the photosynthetic reaction centre from a bacterium, equivalent to a small part of an entire plant reaction centre. In this issue, we are publishing a further step towards the full structural understanding of the plant photosystems, with a $2.6 \AA$ resolution structure of photosystem 1 (PSI) from pea $^{3}$.

This is not the first PSI structure. This group, under the leadership of Nathan Nelson, published a 4.4 A structure in 2003 (ref. 4), and this most recent structure improves on $2.8 \AA$ structures that were published in 2015 (refs 5,6). While this may sound like a small difference, the extra $0.2 \AA$ brings with it visualisation of additional protein, water and lipid components, all contributing to a better understanding of the functioning of this light-driven machine.

Structural biology has never been more important to our understanding of the mechanics of life with constant technical advances bringing with them ever finer acuity. Nature Plants will strive to present the very best of these structural studies with particular relevance to plants; although we won't be changing the journal's name any time soon! $\square$

\footnotetext{
References

1. Hirano, Y. et al. Nat. Plants 3, 17010 (2017).

2. Wang, W. et al. Nat. Plants 3, 17011 (2017).

3. Mazor, Y., Borovikova, A., Caspy, I. \& Nelson, N. Nat. Plants 3, 17014 (2017).

4. Ben-Shem, A., Frolow, F. \& Nelson, N. Nature 426, 630-635 (2003)

5. Mazor, Y., Borovikova, A. \& Nelson, N. eLife 4, e07433 (2015).

6. Qin, X., Suga, M., Kuang, T. \& Shen, J. R. Science

348, 989-995 (2015).
} 